\title{
Insights into the Gut Microbiota of Freshwater Shrimp and Its Associations with the Surrounding Microbiota and Environmental Factors ${ }^{\mathbb{S}}$
}

\author{
Yanting Zhao ${ }^{1 \dagger}$, Cuilan Duan ${ }^{2 \dagger}$, Xu-xiang Zhang ${ }^{1}$, Huangen Chen ${ }^{2}$, Hongqiang Ren ${ }^{1}$, Ying Yin ${ }^{1 *}$, and Lin Ye $^{1 *}$ \\ ${ }^{1}$ State Key Laboratory of Pollution Control and Resource Reuse, School of the Environment, Nanjing University, Nanjing 210023, P.R. China \\ ${ }^{2}$ Fisheries Technology Extension Center of Jiangsu Province, Nanjing 210036, P.R. China
}

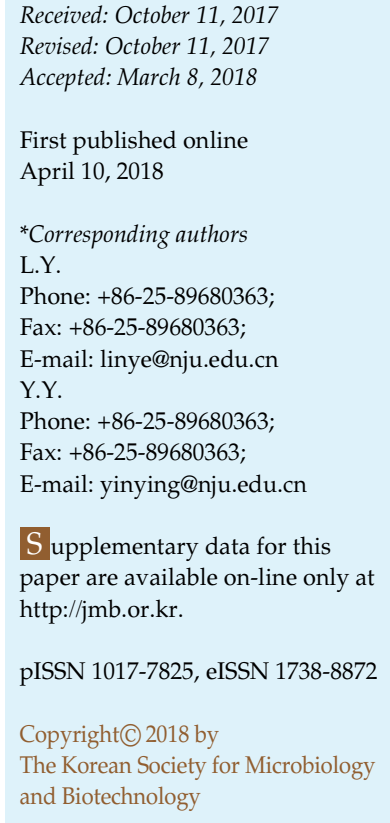

The gut microbiota of aquatic animals plays a crucial role in host health through nutrient acquisition and outcompetition of pathogens. In this study, on the basis of the highthroughput sequencing of 16S rRNA gene amplicons, we examined the bacterial communities in the gut of freshwater shrimp (Macrobrachium nipponense) and in their living environments (sediment and pond water) and analyzed the effects of abiotic and biotic factors on the shrimp gut bacterial communities. High bacterial heterogeneity was observed in the freshwater shrimp gut samples, and the result indicated that both the surrounding bacterial community and water quality factors (particularly dissolved oxygen and temperature) could affect the shrimp gut bacterial community. Despite the observed heterogeneity, 57 genera, constituting $38-99 \%$ of the total genera in each of the 40 shrimp gut samples, were identified as the main bacterial population in the gut of $M$. nipponense. In addition, a high diversity and abundance of lactic acid bacteria (26 genera), which could play significant roles in the digestion process in shrimp, were observed in the shrimp gut samples. Overall, this study provides insights into the gut bacterial communities of freshwater shrimp and basic information for shrimp farming regarding the application of probiotics and disease prevention.

Keywords: Freshwater shrimp, gut microbiota, environmental factors, lactic acid bacteria

\section{Introduction}

Over the past decade, many studies have been conducted to investigate the interaction between gut microbiotas and their hosts [1, 2]. Previous findings have revealed that gut microorganisms are not only associated with animal growth $[3,4]$ but also related to disease phenotypes, such as liver diseases and obesity [5,6]. Substantial work has been carried out to characterize the factors, such as infection status or host phylogeny, that may shape gut microbial communities $[7,8]$. Specifically, the diversity and abundance of gut microbial communities could be significantly affected by environmental factors. For instance, previous research has indicated that house dust plays a role in shaping the nascent gut microbiota [9]. Sullam et al. [10] showed that fish microbial communities are mainly shaped by environmental and ecological factors. The environmental temperature can also influence the gut microbial community structure, especially in amphibians [11]. From an ecological perspective, the gut can be regarded as a unique microbial assemblage, in which microbial diversity and structure can be explained by principles of classical island biogeography theory [12]. Some recent studies have provided evidence that microorganisms display biogeographic patterns, some of which are similar to the large organisms [13, 14]. Yan et al. [15] described the animal gut as an "island" ecosystem that appeared to be mainly shaped by the gut environment and certain other selective changes accompanying the host development process.

To understand the gut microbiome, it is of crucial importance to identify the "core" microbial community and presumably significant organisms [16]. Recently, 
phylogenetically defined core gut microbiotas have been described in bees [17], termites [18], the simple chordate Ciona intestinalis [19], and especially in humans, highlighting significant interindividual and interpopulation variability [20]. Greenhalgh et al. [21] defined baseline healthy microbiomes and resolved microbiome-conferred functionalities associated with human health. However, explicit studies of core communities associated with aquatic animals such as shrimp are still rare.

In recent years, a number of studies aimed at investigating the relationships between aquatic animals and their gut microbiotas have been conducted. It has been reported that bacteria in the intestines of aquatic animals may contribute to the development of host immune systems and digestive systems [22]. Most of the studies on shrimp gut microbiota conducted to date have focused on seawater shrimp. For example, the study on the black tiger shrimp showed that the development of probiotics could enhance growth and disease resistance [23]. Liu et al. [24] investigated the bacterial composition of the intestines of wild-caught and domesticated Penaeus monodon, and their results indicated that the internal environments within host shrimp exert selective pressure on the bacterial community. Additionally, associations between dietary fatty acid profiles and the intestinal bacterial composition of Pacific white shrimp (Litopenaeus vannamei) have been reported [25]. However, a very limited number of studies aimed at investigating the gut microbiota of freshwater shrimp have been conducted to date.

Macrobrachium nipponensis (Crustacea, Decapoda, Palaemonidae), one of the most important freshwater shrimp species for aquaculture, is broadly distributed in various countries around the world [26]. Tzeng et al. [27] performed pairwise comparisons in oriental river prawns and found that host genetics had a greater impact on the divergence of the gut microbiome than host habitats. Although this study provided important information, a great deal about the microbial community of freshwater shrimp guts remains unknown. For example, how do environmental factors affect the shrimp gut microbiota? Is it possible to define a core microbial population for shrimp? Which microorganisms act as probiotics or pathogens in shrimp? Answering these questions may improve our understanding of gut microbial ecology and provide useful information for shrimp farming.

\section{Materials and Methods}

Sample Collection and Analyses of Environmental Parameters
In this study, freshwater shrimps ( $M$. nipponense) were collected from 25 shrimp ponds at six shrimp farms located in Liyang (LY), Yangzhong (YZ), Wuxi (WX), Suzhou (SZ), Xinghua (XH), and Pukou (PK) of Jiangsu Province, China, between March and September of 2016. Information about the sampling sites and shrimp is summarized in Table S1. Sediment and pond water samples from each shrimp pond were collected at the same time. At each farm, 20 disease-free shrimps with nearly consistent size and weight (Table S1) were collected from three different ponds and transported to the laboratory within $4 \mathrm{~h}$ for dissection. A $1 \mathrm{~L}$ sample of pond water was collected from the center of each pond at a depth of $10 \mathrm{~cm}$, and five sediment samples were collected from each shrimp pond (one from the center and the other four from the four corners of each pond). Equal volumes of the five sediment samples from each pond were then mixed to form a single sample (approximately $200 \mathrm{~g}$ ). The gut contents of the shrimp from each pond collected at each sampling time were pooled for DNA extraction. The pond water samples were filtered through a $0.45-\mu \mathrm{m}$-pore-size membrane to collect bacteria. After pretreatment, all of the samples (shrimp gut contents, membrane filtrates, and sediments) were collected and stored at $-80^{\circ} \mathrm{C}$ until DNA extraction.

Total organic carbon (TOC), total nitrogen (TN), and total phosphorus (TP) were analyzed according to standard methods. The water temperature, $\mathrm{pH}$, and dissolved oxygen (DO) were measured with portable meters (Table S2).

\section{DNA Extraction, PCR, and High-Throughput Sequencing of the $16 S$ rRNA Gene}

DNA was extracted from the sediment, gut content, and pond water samples using the FastDNA SPIN Kit for Soil (MP Biomedicals, USA) according to the manufacturer's instructions. The concentration and quality of the extracted DNA were checked using a NanoDrop 2000 device (NanoDrop Technologies, USA) and agarose gel electrophoresis. The primer set 341F (5'CCTACGGGNGGCWGCAG-3') and 806R (5'-GGACTACHVGGG TWTCTAAT-3'), targeting the hypervariable V3 and V4 regions of the $16 \mathrm{~S}$ rRNA gene, was used for PCR amplification. PCR amplification was conducted in a reaction system $(30 \mu \mathrm{l})$ containing $5 \mu \mathrm{l}$ of template DNA $(50 \mathrm{ng} / \mu \mathrm{l}), 2.5 \mu \mathrm{l}$ of the forward primer, $2.5 \mu \mathrm{l}$ of the reverse primer, $5 \mu \mathrm{l}$ of $\mathrm{ddH}_{2} \mathrm{O}$, and $15 \mu \mathrm{l}$ of $2 \times$ Phusion High-Fidelity PCR Master Mix with GC Buffer. The thermocycling steps included an initial denaturation step of $95^{\circ} \mathrm{C}$ for $2 \mathrm{~min}$, followed by 30 cycles of $95^{\circ} \mathrm{C}$ for $20 \mathrm{sec}, 51^{\circ} \mathrm{C}$ for $30 \mathrm{sec}, 72^{\circ} \mathrm{C}$ for $60 \mathrm{sec}$, and a final elongation step at $72^{\circ} \mathrm{C}$ for $5 \mathrm{~min}$. The PCR products were purified with a QIAquick PCR Purification Kit (Qiagen, Germany) and quantified on a Qubit 2.0 Fluorometer (Invitrogen, USA). The purified PCR products were sent to Jiangsu Zhongyijinda Analytical \& Testing Co., Ltd. (China) for library preparation and high-throughput sequencing on the MiSeq platform (Illumina, USA). The sequencing data have been deposited in the Sequence Read Archive under the accession number PRJNA381860. 


\section{Sequencing Data from Other Studies}

Additional shrimp gut bacterial 16S rRNA gene sequences reported in previous studies were retrieved from GenBank for comparative analysis. The detailed information on these sequences is shown in Table S3.

\section{Processing of Sequencing Data and Statistical Analysis}

After sequencing, the paired-end reads were joined using the "make.contigs" command of Mothur [28], and potential chimeric sequences introduced in the PCR process were then detected and removed using "chimera.uchime." The high-quality reads were subsequently employed to cluster preprocessed reads into operational taxonomic units (OTUs) at a similarity of 0.97 using the Quantitative Insights into Microbial Ecology (QIIME) pipeline [29]. Low-abundance OTUs (<three reads) were regarded as sequencing noise and removed from further analysis. The taxonomy of the representative sequence of each OTU was assigned with the RDP Classifier [30]. The differences in the microbial community among different samples were analyzed by nonmetric multidimensional scaling (NMDS) based on Bray-Curtis distance. In addition, redundancy analysis (RDA) was used to reveal the relationships between the water quality parameters and the abundance of the major OTUs. Both NMDS and RDA were performed using the "vegan" package in R (ver. 3.2.3). Variance partitioning analysis (VPA) was employed to determine the contributions of the sediment and pond water microbiotas, as well as interactions between them, to the shrimp gut bacterial communities. Heatmap analysis and VPA were also performed in $\mathrm{R}$ (ver. 3.2.3) with the "gplots" and "vegan" packages. The significantly different taxa in different groups of samples were determined and visualized using linear discriminant analysis effect size (LEfSe), a statistical tool designed to find biomarkers from the data with default parameters [31].

\section{Results and Discussion}

\section{Bacterial Communities in Shrimp Guts, Pond Water, and Sediment}

In this study, the microbial communities in the shrimp guts, pond water, and sediment were analyzed on the basis of 4,452,347 quality-filtered, non-chimeric $16 \mathrm{~S}$ rRNA gene sequences. Fig. 1 illustrates the relative abundance of the major phyla that were commonly observed in the three kinds of samples. In general, the microbial community of the sediment samples showed a higher level of diversity and stability than those of the shrimp guts and pond water. The most dominate phylum in the sediment was Proteobacteria (15.79-52.40\%), followed by Bacteroidetes (2.35-33.34\%), Firmicutes (2.73-26.02\%), Chloroflexi (1.39$16.67 \%)$, and Actinobacteria (1.34-13.80\%). However, the microbial composition observed in the pond water and shrimp guts varied significantly at different sampling times. The three most abundant phyla in pond water were Proteobacteria (14.35-68.30\%), Actinobacteria (2.06-51.08\%), and Bacteroidetes (3.68-50.37\%). Cyanobacteria displaced Proteobacteria as the dominant bacterial phylum in some pond water samples collected in June, July, and September, which could be due to algal blooms in the shrimp ponds from June to September. Proteobacteria was the dominant phylum of the microbial community in the shrimp gut samples collected in March, accounting for $89.68 \pm 1.21 \%$ of the total bacterial population on average. From April to

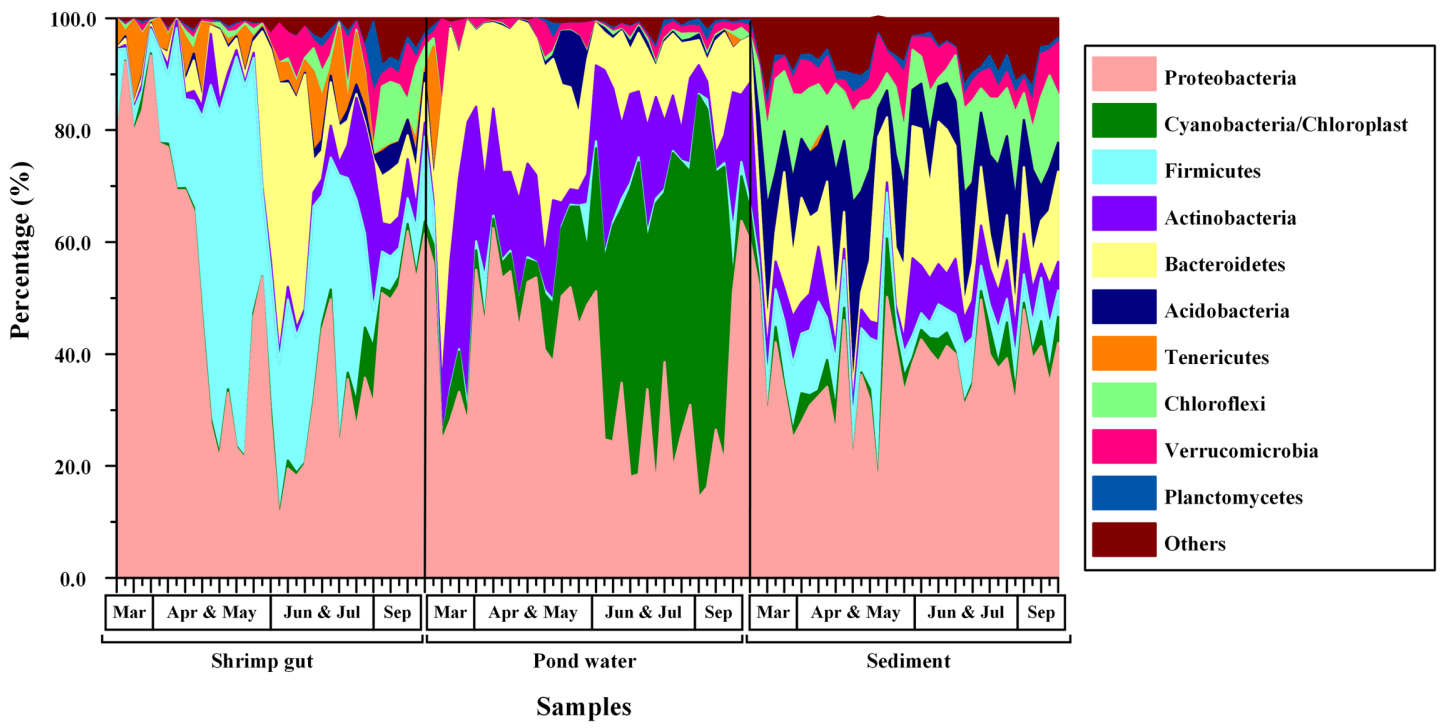

Fig. 1. Bacterial communities in shrimp guts and the surrounding environment (water and sediment) at different sampling times. Sampling was performed separately six times at different sites, in March, April, May, June, July, and September of 2016. 
July, a decrease in Proteobacteria (from $77.66 \pm 0.83 \%$ to $35.51 \pm 1.09 \%$ ) and marked increases in Firmicutes (from $3.89 \pm 1.34 \%$ to $69.65 \pm 1.17 \%$ ) and Bacteroidetes (from $0.53 \pm 0.21 \%$ to $41.89 \pm 1.11 \%$ ) were observed. With the abundant occurrence of Acidobacteria (1.10-26.39\%) and Chloroflexi (0.54-13.29\%), the microbial communities of the gut samples collected in September displayed a higher complexity than those of other samples. Proteobacteria, Firmicutes, Actinobacteria, and Bacteroidetes were also reported as the main bacterial populations found in freshwater shrimp in a previous study [27]. However, our findings differed from results reported for black tiger shrimp, which showed a dominance of Gammaproteobacteria, followed by Alphaproteobacteria, Bacteroidetes, and Firmicutes [32]. The differences in the microbial communities between different shrimp are shown in the NMDS plot in Fig. S1, where it could be seen that the seawater shrimp (L. vannamei and P. monodon) samples were separated from freshwater shrimp samples (M. nipponense) and formed a distinct cluster, which indicated that the differences in the gut microbial communities may be attributed to the hosts or habitats

Moreover, the bacterial diversity and abundance in the shrimp gut, pond water, and sediment samples were analyzed more specifically at the OTU level (3\% dissimilarity). Notably, the 36 abundant OTUs (abundance $>10.00 \%$ in at least one sample) exhibited clear differences between the gut, sediment, and pond water samples (Fig. S2). In the gut samples, the most abundant OTU (representing 10.45 \pm $1.46 \%$ of all bacterial sequences on average) was assigned to the genus Dongia, which has been isolated from soil [33], freshwater [34], and stone biofilms in Lake Constance [35]. In addition, Clostridium XlVb $(5.10 \pm 0.19 \%)$, Aeromonas $(4.52 \pm 1.17 \%)$, Citrobacter $(4.00 \pm 0.36 \%)$, and Bacillariophyta $(1.95 \pm 0.54 \%)$ were other major genera in the shrimp gut samples. Interestingly, Clostridium and Bacillariophyta were also common in freshwater fish [36]. Among these bacterial genera, Aeromonas and Bacillariophyta were identified in a wide range of samples, and their total abundance in the shrimp gut $(6.74 \pm 0.15 \%)$ and pond water samples $(3.88 \pm 0.60 \%)$ was higher than in the sediment samples $(0.08 \pm 0.76 \%)$. In the pond water samples, the dominant OTUs were assigned to Bacillariophyta $(4.57 \pm 0.09 \%)$, Aeromonas $(3.57 \pm 0.12 \%)$, GpI $(1.79 \pm 0.23 \%)$, Acinetobacter $(1.76 \pm 0.32 \%)$, and GpIIa $(1.67 \pm 0.43 \%)$. However, no high-abundance genera were observed in the sediment samples. Bacillariophyta $(0.44 \pm$ $0.36 \%)$, Methylocystis $(0.21 \pm 0.16 \%)$, GpIIa $(0.16 \pm 0.14 \%)$, and Clostridium sensu stricto $(0.10 \pm 0.18 \%)$ were commonly found in sediment samples with low abundance.

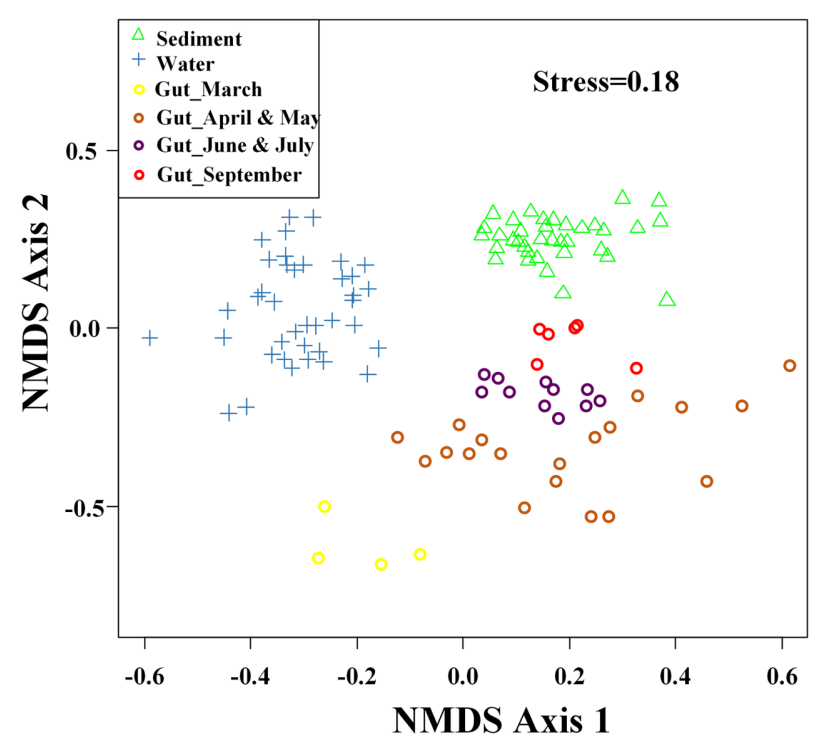

Fig. 2. NMDS plot showing differences in the microbial community among different samples.

The distances were determined using the Bray-Curtis method on the basis of OTU relative abundance.

On the basis of the relative abundance of OTUs, the NMDS plot (Fig. 2), a nonparametric method that provides graphical ordination of the experimental data [37], was employed to compare the similarity of the microbial community composition among different samples. As expected, the three kinds of samples were clearly separated and formed three distinct clusters owing to the significantly different bacterial communities. Despite the variation in the relative abundance of shrimp gut microbes, the bacterial communities in shrimp guts from all sampling times were more close to the bacterial community in the sediment than to that in the pond water. Moreover, as the study period progressed (from March to September), the shrimp gut samples moved towards the cluster of sediment samples in the plot, suggesting that the bacterial community in shrimp guts changed during this process and became more similar to that in the sediment. This result was probably due to the ingestion of some of the bacteria in the sediment by the shrimp, which also suggested that environmental factors could mediate the community structure and that the gut microbiota is habitat-specific [38].

\section{Relationships between the Shrimp Gut Bacterial Community and Environmental Factors}

RDA was used to investigate the relationship between the bacterial community in shrimp guts and environmental factors, including water temperature, $\mathrm{pH}, \mathrm{TP}, \mathrm{DO}, \mathrm{TOC}$, 


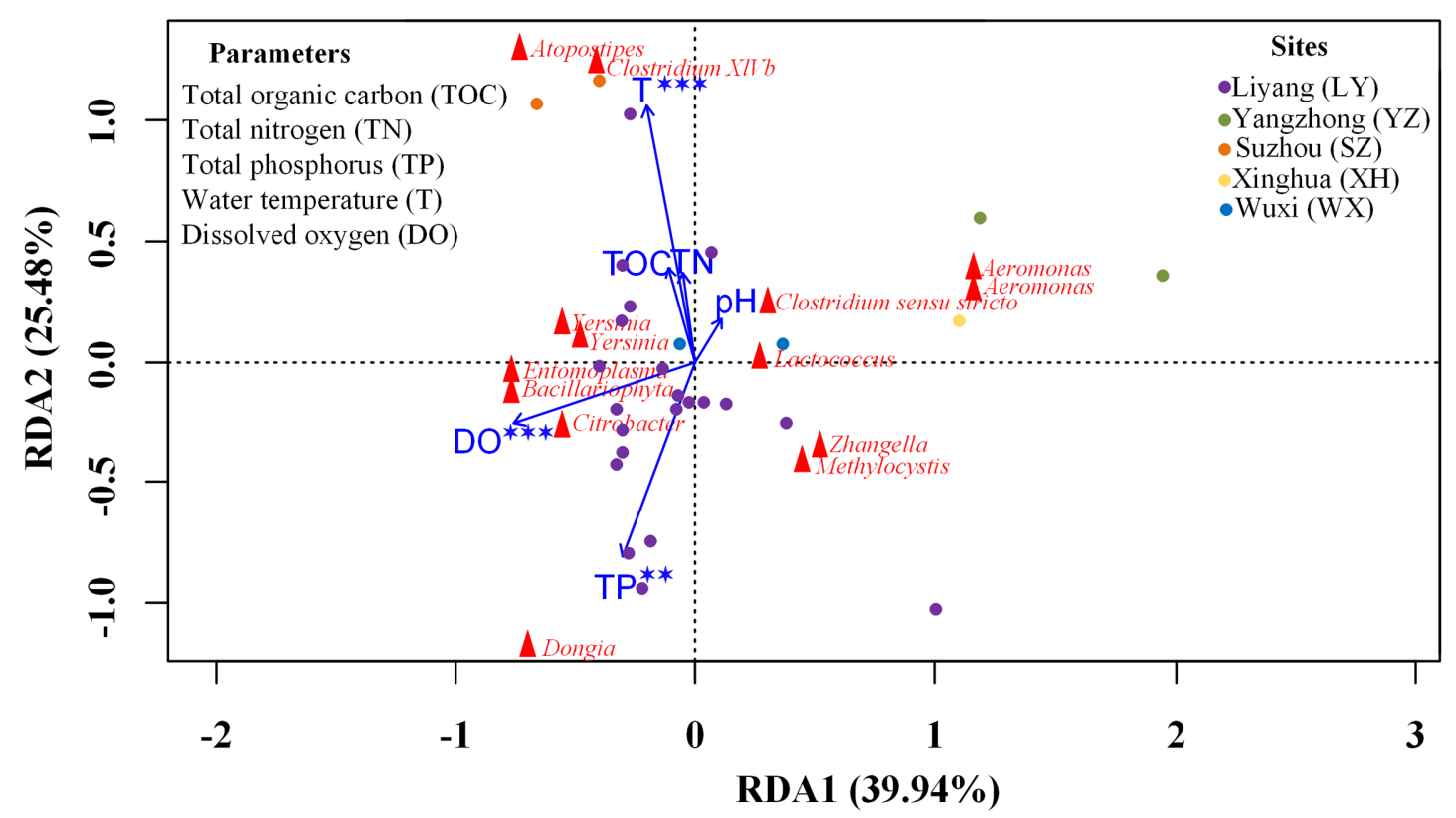

Fig. 3. Redundancy analysis illustrating the relationships of the water quality parameters (TP, TOC, TN, T, DO, and pH) (arrows) with the abundance of the major OTUs (triangular symbols) in the gut samples.

Different colors of the symbols indicate different sampling sites.

and TN. As shown in Fig. 3, three environmental variables, temperature $(p=0.004)$, TP $(p=0.01)$, and DO $(p=0.007)$, were found to significantly contribute to the bacterial community-environment relationship. Many genera in shrimp gut, such as Entomoplasma, Bacillariophyta, and Citrobacter, were positively correlated with DO. Atopostipes and Clostridium $\mathrm{XlVb}$ were positively correlated with temperature. It is widely accepted that temperature is an important factor shaping the bacterial community structure in natural environments [39]; however, its effects on the bacterial communities of animal guts have seldom been reported. Dongia in shrimp gut was the only genus that was positively correlated with TP. As for the different sampling locations, the abundant genera in samples collected from SZ were related to a higher temperature. On the other hand, most of the samples collected from LY and WX were associated with high DO and low temperature, whereas the samples collected from $\mathrm{YZ}$ were separated from the others. These results demonstrated the importance of DO and temperature as the major determinants shaping shrimp gut bacterial communities.

We further investigated the relative contributions of abiotic environmental factors (water quality parameters) and biotic environmental factors (bacterial communities in pond water and sediment) to the shrimp gut bacterial community. VPA was carried out to partition the variations in bacterial community structure between pond water bacterial communities, sediment bacterial communities, and water quality parameters, as well as their combinations. The results (Fig. S3) suggested that these variables could explain $41.19 \%$ of the observed variation, leaving $58.18 \%$ of the variation unexplained. Water quality parameters contributed $18.84 \%$ to the variation in the shrimp gut microbiota, whereas the sediment and pond water bacterial communities had few effects on the gut microbiota composition (negative values were interpreted as zeros). However, the variation could be better explained (36.04\%) by the joint effects of the sediment and pond water bacterial communities. To the best of our knowledge, the roles of water quality, the surrounding bacterial community, and their combination in shaping shrimp gut microbial communities have rarely been reported. Our results indicated that both the surrounding bacterial community and water quality factors might affect the shrimp gut microbial community. A better understanding of the influencing mechanisms could be helpful for establishing new microbial management strategies to improve aquatic food production [40].

\section{Main Bacterial Population in Shrimp Guts}

Identifying the dominant population is conducive to better understanding the ecology of the shrimp gut 
microbial community. To determine the core bacterial community, we compared 40 shrimp gut samples from six shrimp farms. However, only two OTUs were observed in every sample, and accounted for only $7.44 \%$ of the total reads. Therefore, it is difficult to define a core bacterial population shared by all shrimp guts owing to the heterogeneity of the microbial community. In this study, to characterize the dominant bacteria in shrimp guts, we identified the main bacterial genera on the basis of the OTU abundance and frequency.

As shown in Fig. 4A, the more frequently observed genera tended to be more abundant. The genera observed in 32 or more samples accounted for $51.28-99.48 \%$ of the total reads, whereas the 429 genera observed in five or fewer samples accounted for only $0.52 \%$ of the total reads. In this study, we defined 57 genera (Fig. 4B) as the shared main gut bacterial population on the basis of frequency (>32 samples) and abundance ( $>1.0 \%$ in at least one sample). These genera accounted for $38.00-99.48 \%$ of the shrimp gut samples, with only two exceptions, which might have been due to infection by pathogenic bacteria (Fig. 4C). It was found that the potential pathogenic bacteria belonging to Yersinia and Lawsonia accounted for $92.01 \%$ and $81.97 \%$ of these two samples, respectively (Fig. 4C). At the phylum level, the four most abundant taxa were Proteobacteria, Firmicutes, Bacteroidetes, and Acidobacteria, which showed an abundance ranging from 2.44-95.07\%, 0.18-65.02\%, $0.01-35.76 \%$, and $0.01-10.49 \%$, respectively.

The high abundance of the 57 main genera in freshwater shrimp supports the idea that the main microbiota of healthy individuals will be fundamentally composed of coevolved beneficial microbes, rather than randomly assorted ones [41]. A number of these bacteria have been functionally characterized, and many of them are important for shrimp growth as probiotics. For example, Lactobacillus (accounting for $10.06 \pm 1.71 \%$ on average) played an important role in the physiology of their hosts by providing a protective barrier in the gut, and it has been reported that the extensive use of Lactobacillus as a feed additive for poultry can reduce infections caused by intestinal pathogens [42]. Citrobacter $(4.95 \pm 1.09 \%)$ cellulose-degrading bacteria have been isolated from the gastrointestinal tract of herbivorous fish species and shown to be able to metabolize a remarkable variety of substrates, including fibrins in diets [43]. In addition to the Citrobacter genus, Acinetobacter $(0.14 \pm 1.10 \%)$, Aeromonas $(6.97 \pm 1.22 \%)$, Flavobacterium $(0.61 \pm 0.18 \%)$, Pseudomonas (0.66 $\pm 0.26 \%$ ), and Bacillus ( $0.24 \pm 1.42 \%$ ) have been suggested as possible contributors to cellulolytic enzyme production [44]. In addition, Bacteroides was included in the 57 main genera, with a relatively low abundance $(0.35 \%)$. Bacteroides may play a fundamental role in the processing of complex molecules in animal guts [45].

\section{High Diversity and Abundance of Lactic Acid Bacteria in Shrimp Guts}

It is widely accepted that lactic acid bacteria (LAB) are important to the digestion systems of animals, and these bacteria have been intensively applied as probiotic supplements to enhance immunity and disease resistance in aquaculture [46-48]. In this study, we investigated the diversity and abundance of LAB in the shrimp gut samples. Fig. S4 shows that 26 genera of LAB were identified in the shrimp gut samples, belonging to Streptococcaceae (4.64 \pm $1.32 \%)$, Carnobacteriaceae $(3.62 \pm 0.98 \%)$, Aerococcaceae $(0.14 \pm 0.83 \%)$, Lactobacillaceae $(0.62 \pm 1.15 \%)$, Enterococcaceae $(0.10 \pm 0.93 \%)$, and Leuconostocaceae $(0.01 \pm 0.13 \%)$, which collectively constituted up to $56.25 \%$ of the total genera in the shrimp guts. Among these taxa, Lactobacillus and Lactococcus were the major LAB in shrimp guts, accounting for proportions of up to $50.34 \%$ and $3.72 \%$, respectively.

Moreover, we compared the LAB community in the guts of different shrimp species. The results presented in Fig. S5 indicate that LAB show higher abundance in freshwater shrimp than seawater shrimp. Interestingly, we also noted that the Atopobacter genus was highly host associated and was absent from seawater shrimp (L. vannamei and P. monodon), whereas Tetragenococcus, Leuconostoc, and Granulicatella were found in only seawater shrimp guts. In addition, Streptococcus is one of the major LAB genera found in L. vannamei and P. monodon. It has been reported that Leuconostoc could reside in the Oncorhynchus keta intestine and showed cholesterol-lowering effects, suggesting its potential use as a fish probiotic $[49,50]$.

In addition, comparisons of the shrimp gut microbiotas with those in the surrounding environments revealed that the shrimp gut microbiotas differed greatly from the environmental microbiotas, and this difference was largely reflected in LAB (Fig. S6). Lactobacillales played a beneficial role in the host gut environment by producing antibacterial substances, such as lactic acid, acetic acid, hydrogen peroxide, and bacteriocin, and by inhibiting increases in harmful intestinal bacteria [51]. Enterobacteriaceae, which were regarded as enterobacteria or "enteric bacteria" and live in the intestines of animals, are facultative anaerobes that ferment sugars to produce lactic acid and various other end products [52]. 

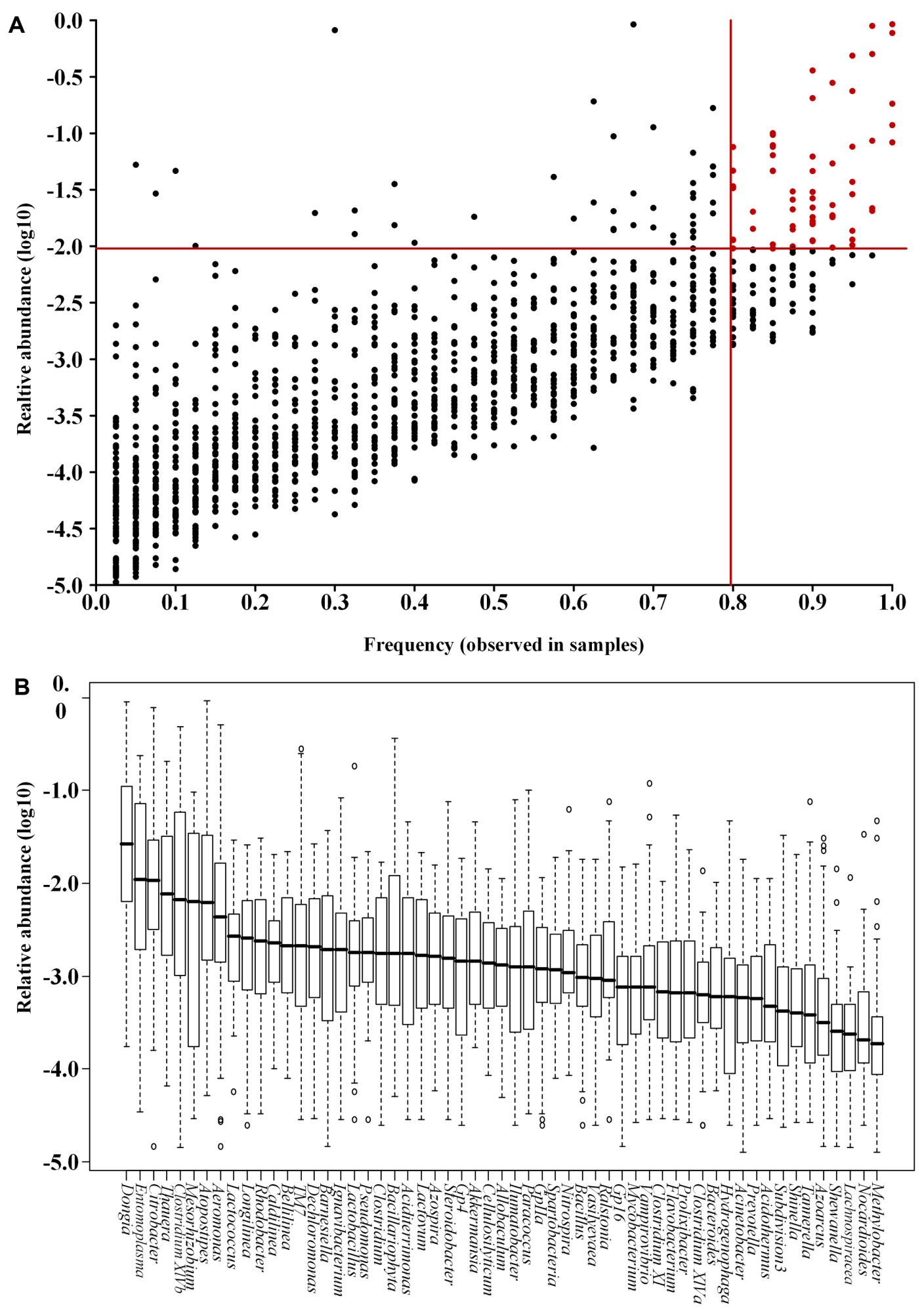

Fig. 4. Frequency of occurrence and relative abundance of the bacterial genera in shrimp gut samples.

(A) Relationships between the frequency of occurrence and relative abundance of the bacterial genera. (B) Box plot showing the relative abundance of the 57 main bacteria among individual shrimp gut samples. (C) Relative abundance of the 57 main bacterial genera in each of the 40 shrimp gut samples. 


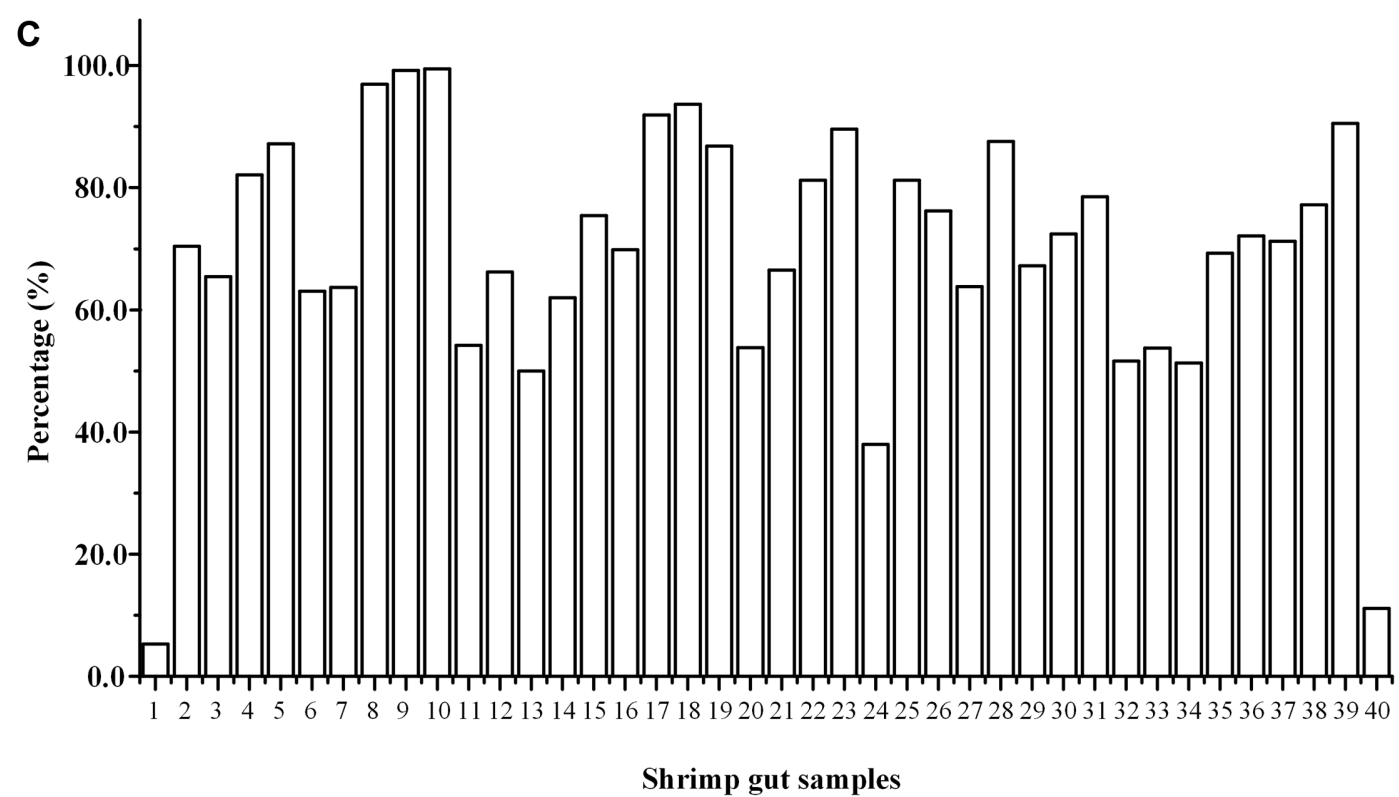

Fig. 4. Continued.

\section{Potential Pathogenic Bacteria in Shrimp Guts}

The animal gut is considered a main route of pathogen transmission [53]; therefore, understanding which pathogenic bacteria is of great importance for shrimp farming. In this study, the investigation of opportunistic pathogens revealed certain potential health risks in shrimp and their living environment. Ten potentially pathogenic genera that have been reported in the previous literature are shown in Table S4. The following major opportunistic pathogenic genera were detected in the shrimp guts: Aeromonas (up to $6.97 \%)$, Yersinia (4.49\%), Pseudomonas (0.66\%), Mycobacterium $(0.62 \%)$ and Flavobacterium $(0.61 \%)$. This finding is generally consistent with those of a previous study on oriental river prawns [27]. Among these genera, Aeromonas caviae, Aeromonas hydrophila, and Mycobacterium marinum were the most common pathogens related to aquatic animals, including shrimp [54], whereas Mycobacterium and Flavobacterium exist in various environments, including freshwater, and can cause a series of complex diseases, including Mycobacterium avium complex disease [55-57]. Previous studies revealed that Vibrionaceae-related microorganisms, which are virtually ubiquitous in aquatic environments, cause the most economically serious diseases, such as vibriosis [58-60]. However, it was noted that Vibrio accounted for only a small proportion $(0.03 \%)$ of the freshwater shrimp pathogens compared with that in seawater shrimp (11.45\%) (Fig. S7).

According to the findings described herein and previous studies, detrimental opportunistic pathogens are a normal phenomenon in aquatic animal production. Controlling diseases in aquaculture animals by using a wide variety of antibiotics in large amounts was responsible for a growing problem related to both human and animal health as well as the environment [53]. Therefore, researchers have highlighted the importance of ecological theory to shape the gut microbiota for disease control in aquaculture [40, 61]. Several studies have illustrated the manipulation of intestinal microbiota using probiotics, rather than prebiotics [23, 62]. Attramadal et al. [63, 64] indicated that the reared microbiota may be managed toward a state in which the contact between opportunistic pathogens and cultured animals is minimized, thus decreasing the disease risk for the cultured animals. Furthermore, a new pathogen management strategy was proposed to manage the water microbiota in aquaculture systems according to ecological selection principles in order to decrease the pressure from opportunistic pathogens on aquatic animals [40]. In summary, an ecological perspective may greatly contribute to disease prevention and control in aquaculture animal production. Further studies are needed to investigate the detailed underlying mechanisms and to develop practical approaches.

In conclusion, the results of this study revealed high bacterial heterogeneity in freshwater shrimp guts, and Proteobacteria was found to be the most abundant bacterial group in freshwater gut samples (accounting for $51.40 \pm$ $1.92 \%$ on average), which significantly differs from the 
results reported in seawater shrimp. The most abundant OTU (representing $10.45 \pm 1.46 \%$ of all bacterial sequences on average) was assigned to the genus Dongia. Despite the high observed heterogeneity, associations of the shrimp gut bacterial community with water quality parameters (such as DO and temperature) and the bacterial populations in the sediment and pond water were observed. Further analyses of the gut microbiotas revealed 57 main genera and a high diversity and abundance of LAB in the shrimp gut. Moreover, some potentially pathogenic genera were identified. Future research should focus on the functions of the bacteria in the shrimp gut to provide information for establishing sustainable microbial management strategies for shrimp farming.

\section{Acknowledgments}

This work was supported by the Jiangsu Agriculture Science and Technology Innovation Fund (CX(15)1012-3), the Fundamental Research Funds for the Central Universities of China (14380034), and the State Key Program of National Natural Science of China (41230640).

\section{Conflict of Interest}

The authors have no financial conflicts of interest to declare.

\section{Ethical Approval}

Animal ethics approval for the present project was obtained from the Animal Ethics Committee of Fisheries Technology Extension Center of Jiangsu Province, China.

\section{References}

1. Backhed F. 2011. Programming of host metabolism by the gut microbiota. Ann. Nutr. Metab. 58: 44-52.

2. Ley RE, Hamady M, Lozupone C, Turnbaugh PJ, Ramey RR, Bircher JS, et al. 2008. Evolution of mammals and their gut microbes. Science 320: 1647-1651.

3. Angelakis E, Armougom F, Million M, Raoult D. 2012. The relationship between gut microbiota and weight gain in humans. Future Microbiol. 7: 91-109.

4. Khan I, Yasir M, Azhar EI, Kumosani T, Barbour EK, Bibi F, et al. 2014. Implication of gut microbiota in human health. CNS Neurol. Disord. Drug Targets 13: 1325-1333.

5. Schnabl B, Brenner DA. 2014. Interactions between the intestinal microbiome and liver diseases. Gastroenterology 146: 1513-1524.
6. Grigorescu I, Dumitrascu DL. 2016. Implication of gut microbiota in diabetes mellitus and obesity. Acta Endocrinol. (Bucharest) 12: 206-214.

7. Moeller AH, Li Y, Ngole EM, Ahuka-Mundeke S, Lonsdorf EV, Pusey AE, et al. 2014. Rapid changes in the gut microbiome during human evolution. Proc. Natl. Acad. Sci. USA 111: 16431-16435.

8. Jacobs J, Braun J. 2014. Host genes and their effect on the intestinal microbiome garden. Genome Med. 6: 119.

9. Konya T, Koster B, Maughan H, Escobar M, Azad MB, Guttman DS, et al. 2014. Associations between bacterial communities of house dust and infant gut. Environ. Res. 131: 25-30.

10. Sullam KE, Essinger SD, Lozupone CA, O'Connor MP, Rosen GL, Knight R, et al. 2012. Environmental and ecological factors that shape the gut bacterial communities of fish: a meta-analysis. Mol. Ecol. 21: 3363-3378.

11. Kohl KD, Yahn J. 2016. Effects of environmental temperature on the gut microbial communities of tadpoles. Environ. Microbiol. 18: 1561-1565.

12. Costello EK, Stagaman K, Dethlefsen L, Bohannan BJM, Relman DA. 2012. The application of ecological theory toward an understanding of the human microbiome. Science 336: $1255-1262$.

13. Hanson CA, Fuhrman JA, Horner-Devine MC, Martiny JBH. 2012. Beyond biogeographic patterns: processes shaping the microbial landscape. Nat. Rev. Microbiol. 10: 497-506.

14. Martiny JBH, Bohannan BJM, Brown JH, Colwell RK, Fuhrman JA, Green JL, et al. 2006. Microbial biogeography: putting microorganisms on the map. Nat. Rev. Microbiol. 4: $102-112$.

15. Yan Q, Li J, Yu Y, Wang J, He Z, Van Nostrand JD, et al. 2016. Environmental filtering decreases with fish development for the assembly of gut microbiota. Environ. Microbiol. 18: 4739-4754.

16. Gibson DJ, Ely JS, Collins SL. 1999. The core-satellite species hypothesis provides a theoretical basis for Grime's classification of dominant, subordinate, and transient species. J. Ecol. 87: 1064-1067.

17. Cariveau DP, Powell JE, Koch H, Winfree R, Moran NA. 2014. Variation in gut microbial communities and its association with pathogen infection in wild bumble bees (Bombus). ISME J. 8: 2369-2379.

18. Otani S, Mikaelyan A, Nobre T, Hansen LH, Kone NGA, Sorensen SJ, et al. 2014. Identifying the core microbial community in the gut of fungus-growing termites. Mol. Ecol. 23: 4631-4644.

19. Dishaw LJ, Flores-Torres J, Lax S, Gemayel K, Leigh B, Melillo D, et al. 2014. The gut of geographically disparate Ciona intestinalis harbors a core microbiota. PLoS One 9: e93386.

20. Wong ACN, Chaston JM, Douglas AE. 2013. The inconstant gut microbiota of Drosophila species revealed by $16 \mathrm{~S}$ rRNA 
gene analysis. ISME J. 7: 1922-1932.

21. Greenhalgh K, Meyer KM, Aagaard KM, Wilmes P. 2016. The human gut microbiome in health: establishment and resilience of microbiota over a lifetime. Environ. Microbiol. 18: $2103-2116$

22. Engel P, Moran NA. 2013. The gut microbiota of insects diversity in structure and function. FEMS Microbiol. Rev. 37: 699-735.

23. Balcazar JL, de Blas I, Ruiz-Zarzuela I, Cunningham D, Vendrell D, Muzquiz JL. 2006. The role of probiotics in aquaculture. Vet. Microbiol. 114: 173-186.

24. Liu H, Liu M, Wang B, Jiang K, Jiang S, Sun S, et al. 2010. PCR-DGGE analysis of intestinal bacteria and effect of Bacillus spp. on intestinal microbial diversity in kuruma shrimp (Marsupenaeus japonicus). Chin. J. Oceanol. Limnol. 28: 808-814.

25. Rungrassamee W, Klanchui A, Maibunkaew S, Chaiyapechara S, Jiravanichpaisal P, Karoonuthaisiri N. 2014. Characterization of intestinal bacteria in wild and domesticated adult black tiger shrimp (Penaeus monodon). PLoS One 9: e91853.

26. Rahman NMA, Fu HT, Sun SM, Qiao H, Jin S, Bai HK, et al. 2016. Molecular cloning and expression pattern of oriental river prawn (Macrobrachium nipponense) nitric oxide synthase. Genet. Mol. Res. 15: DOI: 10.4238/gmr.15038541.

27. Tzeng T-D, Pao Y-Y, Chen P-C, Weng FC-H, Jean WD, Wang D. 2015. Effects of host phylogeny and habitats on gut microbiomes of oriental river prawn (Macrobrachium nipponense). PLoS One 10: e0132860.

28. Schloss PD, Westcott SL, Ryabin T, Hall JR, Hartmann M, Hollister EB, et al. 2009. Introducing mothur: open-source, platform-independent, community-supported software for describing and comparing microbial communities. Appl. Environ. Microbiol. 75: 7537-7541.

29. Caporaso JG, Kuczynski J, Stombaugh J, Bittinger K, Bushman FD, Costello EK, et al. 2010. QIIME allows analysis of high-throughput community sequencing data. Nat. Methods 7: 335-336.

30. Wang Q, Garrity GM, Tiedje JM, Cole JR. 2007. Naive Bayesian classifier for rapid assignment of rRNA sequences into the new bacterial taxonomy. Appl. Environ. Microbiol. 73: 5261-5267.

31. Jiang X-T, Peng X, Deng G-H, Sheng H-F, Wang Y, Zhou H-W, et al. 2013. Illumina sequencing of $16 \mathrm{~S}$ rRNA tag revealed spatial variations of bacterial communities in a mangrove wetland. Microb. Ecol. 66: 96-104.

32. Rungrassamee W, Klanchui A, Chaiyapechara S, Maibunkaew S, Tangphatsornruang S, Jiravanichpaisal P, et al. 2013. Bacterial population in intestines of the black tiger shrimp (Penaeus monodon) under different growth stages. PLoS One 8: e60802.

33. Kim D-U, Lee H, Kim H, Kim S-G, Ka J-O. 2016. Dongia soli sp. nov., isolated from soil from Dokdo, Korea. Antonie Van Leeuwenhoek 109: 1397-1402.
34. Baik KS, Hwang YM, Choi J-S, Kwon J, Seong CN. 2013. Dongia rigui sp. nov., isolated from freshwater of a large wetland in Korea. Antonie Van Leeuwenhoek 104: 1143-1150.

35. Rahalkar M, Bahulikar RA, Deutzmann JS, Kroth PG, Schink B. 2012. Elstera litoralis gen. nov., sp nov., isolated from stone biofilms of Lake Constance, Germany. Int. J. Syst. Evol. Microbiol. 62: 1750-1754.

36. Ye L, Amberg J, Chapman D, Gaikowski M, Liu W-T. 2014. Fish gut microbiota analysis differentiates physiology and behavior of invasive Asian carp and indigenous American fish. ISME J. 8: 541-551.

37. Prewitt L, Kang Y, Kakumanu ML, Williams M. 2014. Fungal and bacterial community succession differs for three wood types during decay in a forest soil. Microb. Ecol. 68: 212-221.

38. Bletz MC, Goedbloed DJ, Sanchez E, Reinhardt T, Tebbe CC, Bhuju S, et al. 2016. Amphibian gut microbiota shifts differentially in community structure but converges on habitat-specific predicted functions. Nat. Commun. 7: 13699.

39. Staley C, Gould TJ, Wang P, Phillips J, Cotner JB, Sadowsky MJ. 2015. Species sorting and seasonal dynamics primarily shape bacterial communities in the Upper Mississippi River. Sci. Total Environ. 505: 435-445.

40. De Schryver P, Vadstein O. 2014. Ecological theory as a foundation to control pathogenic invasion in aquaculture. ISME J. 8: 2360-2368.

41. Berg M, Stenuit B, Ho J, Wang A, Parke C, Knight M, et al. 2016. Assembly of the Caenorhabditis elegans gut microbiota from diverse soil microbial environments. ISME J. 10: 1998-2009.

42. Dec M, Puchalski A, Nowaczek A, Wernicki A. 2016. Antimicrobial activity of Lactobacillus strains of chicken origin against bacterial pathogens. Int. Microbiol. 19: 57-67.

43. Liu H, Guo X, Gooneratne R, Lai R, Zeng C, Zhan F, et al. 2016. The gut microbiome and degradation enzyme activity of wild freshwater fishes influenced by their trophic levels. Sci. Rep. 6: 24340.

44. Ray AK, Ghosh K, Ringo E. 2012. Enzyme-producing bacteria isolated from fish gut: a review. Aquac. Nutr. 18: 465-492.

45. Xu J, Mahowald MA, Ley RE, Lozupone CA, Hamady M, Martens EC, et al. 2007. Evolution of symbiotic bacteria in the distal human intestine. PLoS Biol. 5: 1574-1586.

46. Dawood MAO, Koshio S, Ishikawa M, Yokoyama S, El Basuini MF, Hossain MS, et al. 2016. Effects of dietary supplementation of Lactobacillus rhamnosus or/and Lactococcus lactis on the growth, gut microbiota and immune responses of red sea bream, Pagrus major. Fish Shellfish Immunol. 49: 275-285.

47. Dimitroglou A, Merrifield DL, Carnevali O, Picchietti S, Avella M, Daniels C, et al. 2011. Microbial manipulations to improve fish health and production - a Mediterranean perspective. Fish Shellfish Immunol. 30: 1-16.

48. Geng X, Dong XH, Tan BP, Yang QH, Chi SY, Liu HY, et al. 2012. Effects of dietary probiotic on the growth performance, 
non-specific immunity and disease resistance of cobia, Rachycentron canadum. Aquac. Nutr. 18: 46-55.

49. Kuda T, Masuko Y, Kawahara M, Kondo S, Nemoto M, Nakata T, et al. 2016. Bile acid-lowering properties of Lactobacillus plantarum Sanriku-SU3 isolated from Japanese surfperch fish. Food Biosci. 14: 41-46.

50. Kuda T, Noguchi Y, Ono M, Takahashi H, Kimura B, Kamita R, et al. 2014. In vitro evaluation of the fermentative, antioxidant, and anti-inflammation properties of Lactococcus lactis subsp. lactis BF3 and Leuconostoc mesenteroides subsp. mesenteroides BF7 isolated from Oncorhynchus keta intestines in Rausu, Japan. J. Funct. Foods 11: 269-277.

51. Russo P, Iturria I, Luz Mohedano M, Caggianiello G, Rainieri S, Fiocco D, et al. 2015. Zebrafish gut colonization by mCherry-labelled lactic acid bacteria. Appl. Microbiol. Biotechnol. 99: 3479-3490.

52. Liu J, Yan Q, Luo F, Shang D, Wu D, Zhang H, et al. 2015. Acute cholecystitis associated with infection of Enterobacteriaceae from gut microbiota. Clin. Microbiol. Infect. 21: 851.e19.

53. Cabello FC. 2006. Heavy use of prophylactic antibiotics in aquaculture: a growing problem for human and animal health and for the environment. Environ. Microbiol. 8: 1137-1144.

54. Tang Y, Tao P, Tan J, Mu H, Peng L, Yang D, et al. 2014. Identification of bacterial community composition in freshwater aquaculture system farming of Litopenaeus vannamei reveals distinct temperature-driven patterns. Int. J. Mol. Sci. 15: 13663-13680.

55. Montiel Quezel-Guerraz N, Marin Arriaza M, Carrillo Avila JA, Sanchez-Yebra Romera WE, Martinez-Lirola MJ, Indal TBG. 2010. Evaluation of the Speed-oligo (R) Mycobacteria assay for identification of Mycobacterium spp. from fresh liquid and solid cultures of human clinical samples. Diagn.
Microbiol. Infect. Dis. 68: 123-131.

56. Abass NA, Suleiman KM, El Jalii IM. 2010. Differentiation of clinical Mycobacterium tuberculosis complex isolates by their GyrB polymorphism. Indian J. Med. Microbiol. 28: 26-29.

57. Manfredi R, Nanetti A, Ferri M, Mastroianni A, Coronado OV, Chiodo F. 1999. Flavobacterium spp. organisms as opportunistic bacterial pathogens during advanced HIV disease. J. Infect. 39: 146-152.

58. Toranzo AE, Magarinos B, Romalde JL. 2005. A review of the main bacterial fish diseases in mariculture systems. Aquaculture 246: 37-61.

59. Tall A, Teillon A, Boisset C, Delesmont R, Touron-Bodilis A, Hervio-Heath D. 2012. Real-time PCR optimization to identify environmental Vibrio spp. strains. J. Appl. Microbiol. 113: $361-372$.

60. Rungrassamee W, Klanchui A, Maibunkaew S, Karoonuthaisiri N. 2016. Bacterial dynamics in intestines of the black tiger shrimp and the Pacific white shrimp during Vibrio harveyi exposure. J. Invertebr. Pathol. 133: 12-19.

61. Xiong J, Dai W, Li C. 2016. Advances, challenges, and directions in shrimp disease control: the guidelines from an ecological perspective. Appl. Microbiol. Biotechnol. 100: 6947-6954.

62. Ringo E, Olsen RE, Gifstad TO, Dalmo RA, Amlund H, Hemre GI, et al. 2010. Prebiotics in aquaculture: a review. Aquac. Nutr. 16: 117-136.

63. Attramadal KJK, Thi My Hanh T, Bakke I, Skjermo J, Olsen Y, Vadstein O. 2014. RAS and microbial maturation as tools for $\mathrm{K}$-selection of microbial communities improve survival in cod larvae. Aquaculture 432: 483-490.

64. Attramadal KJK, Salvesen I, Xue R, Oie G, Storseth TR, Vadstein $\mathrm{O}$, et al. 2012. Recirculation as a possible microbial control strategy in the production of marine larvae. Aquac. Eng. 46: 27-39. 